\title{
Use of Contrast-Enhanced CT in the Diagnosis of Abscesses in Cattle
}

\author{
Ki-Ja LEE ${ }^{1,2)}$, Miori KISHIMOTO ${ }^{2)}$, Junichiro SHIMIZU ${ }^{1,2)}$, Yoshiyasu KOBAYASHI ${ }^{2)}$, Kotaro MATSUMOTO ${ }^{2)}$, \\ Naoki SASAKI ${ }^{1,2)}$, Mitsuo ISHII ${ }^{1,2)}$, Hisashi INOKUMA ${ }^{1,2)}$, Toshiroh IWASAKI ${ }^{1,3)}$, Yoh-Ichi MIYAKE ${ }^{2)}$ and \\ Kazutaka YAMADA ${ }^{1,2)^{*}}$
}

${ }^{1)}$ The United Graduate School of Veterinary Sciences, Gifu University, Gifu 501-1193, 2)Department of Clinical Veterinary Science, Obihiro University of Agriculture and Veterinary Medicine, Obihiro, Hokkaido 080-8555 and ${ }^{3)}$ Department of Veterinary Internal Medicine, Tokyo University of Agriculture and Technology, Tokyo 183-8509, Japan

(Received 31 May 2010/Accepted 8 August 2010/Published online in J-STAGE 23 August 2010)

ABSTRACT. We report here the non-contrast and contrast-enhanced computed tomography performed in two calves with brain abscess and multiple pulmonary abscesses with pharyngeal abscess, respectively. Contrast-enhanced computed tomography was useful in the diagnosis of these diseases in both calves. The diseases were confirmed by histopathological examination. KEY WORDS: abscess, cattle, contrast-enhanced CT, diagnosis.

The number of case reports involving diagnosis with computed tomography (CT) in both companion and production animals have been increasing. At the same time, there are numerous reports on the diagnostic value of contrast-enhanced $\mathrm{CT}$ in small animal practice $[6,7]$. In production animals, however, there have been two reports detailing diagnosis by contrast-enhanced CT in goats [3] and cattle [5]. We wish to report on the importance of contrast-enhanced CT in production animals by detailing two cases diagnosed by this method in cattle.

Case 1 presented with a 5-day history of astasia. On neurological examination, the mental status, papillary light reflex, and menace response were normal. An ataxia of all limbs and opisthotonus was observed. Spinal reflex, panniculus reflex, anal reflex, and pain perception were adequate. Complete blood cell count and serum biochemistry profiles were within normal limits except for leukocytosis $(22,100$ cells $/ \mu \mathrm{L}$, reference range 5,000 $12,000$ cells $/ \mu \mathrm{L})$ consisted of neutrophils $(76 \%)$ and lymphocytes $(22 \%)$. Cerebrospinal fluid (CSF) examination showed leukocytosis $(1,100$ cells $/ \mu \mathrm{L}$, reference range $<25$ cells $/ \mu \mathrm{L})$ and cytology revealed neutrophils (100\%). Based on neurological and CSF examination findings, inflammatory disease in the cerebellum and/or brainstem was suspected.

CT images were obtained by multidetector-row CT (Asteion Super 4, Toshiba, Japan) with the calf under general anesthesia. The calf was anesthetized with $14 \mathrm{mg} /$ $\mathrm{kg}$ pentobarbital sodium (Somnopentyl, Kyoritsu Seikyo Corp., Japan) through the left jugular vein. The calf was positioned in sternal recumbency on the CT table. Contiguous CT scans were obtained with $135 \mathrm{kV}, 150 \mathrm{~mA}$, and $2.0 \mathrm{~mm}$ slice thickness of the head. CT data were reconstructed using an image-processing workstation

\footnotetext{
* Correspondence to: Yamada, K., Department of Clinical Veterinary Science, Obihiro University of Agriculture and Veterinary Medicine, Obihiro, Hokkaido 080-8555, Japan. e-mail:kyamada@obihiro.ac.jp
}

(Virtual Place Advance, AZE, Japan), and transverse and sagittal images were obtained. In non-contrast $\mathrm{CT}$ with a brain window (WL (window level) 100, WW (window width) 80), no abnormal findings were observed (Fig. 1a and c). Sodium iothalamate (Conray, Daiichi Sankyo, Japan) at $600 \mathrm{mgI} / \mathrm{kg}$ body weight was injected into the jugular vein using an autoinjector (Autoenhance A-60, Nemoto Kyorindo, Japan). Post-contrast CT images showed a hypodense (27.0 Hounsfield units) round mass $(1.4 \times 1.4 \mathrm{~cm})$ with ring enhancement in the cerebellum that indicated an abscess (Fig. 1b and d). The calf was euthanized after the CT examination because we considered the prognosis to be poor due to the presence of a brain

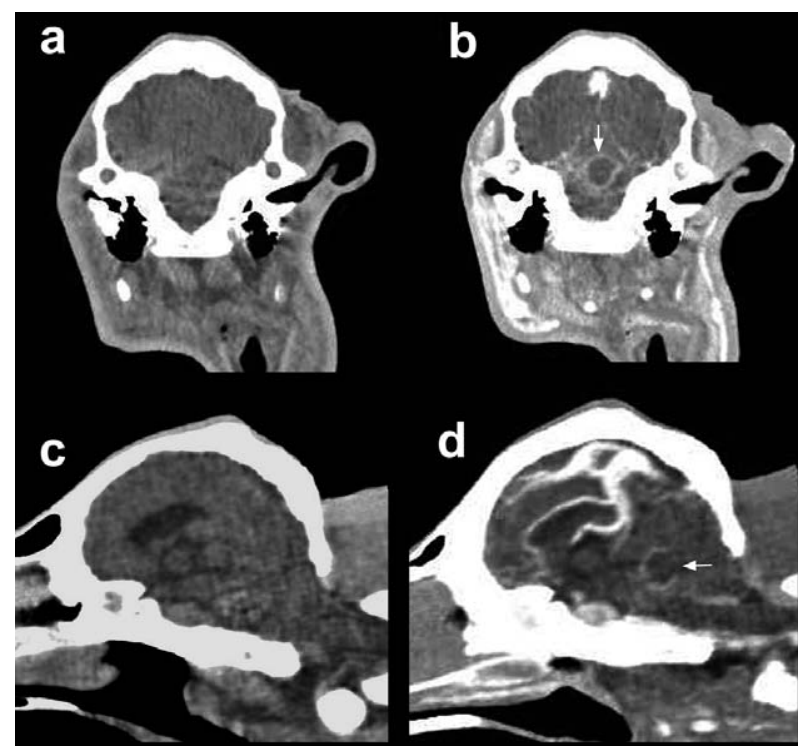

Fig. 1. Transverse $(a, b)$ and sagittal (c, d) CT images of calf with brain abscess. No specific findings observed in noncontrast (a, c) CT images. Post-contrast CT images of the brain show ring enhancement ( $b$ and $d$, arrows), indicating an abscess in the ventral part of the cerebellum. 


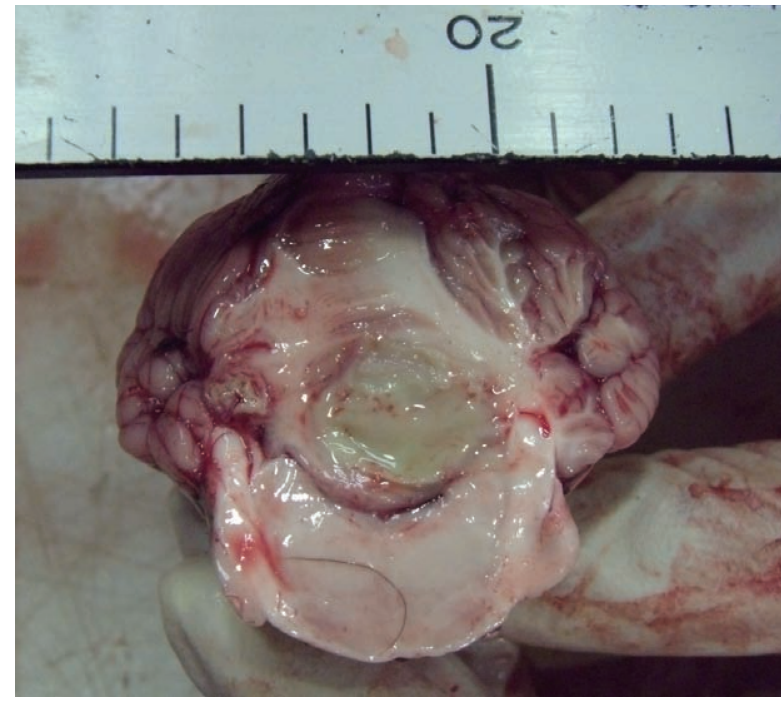

Fig. 2. Transverse section at the level of cerebellum in Case 1 shows the abscess in the ventral region of the cerebellar vermis.

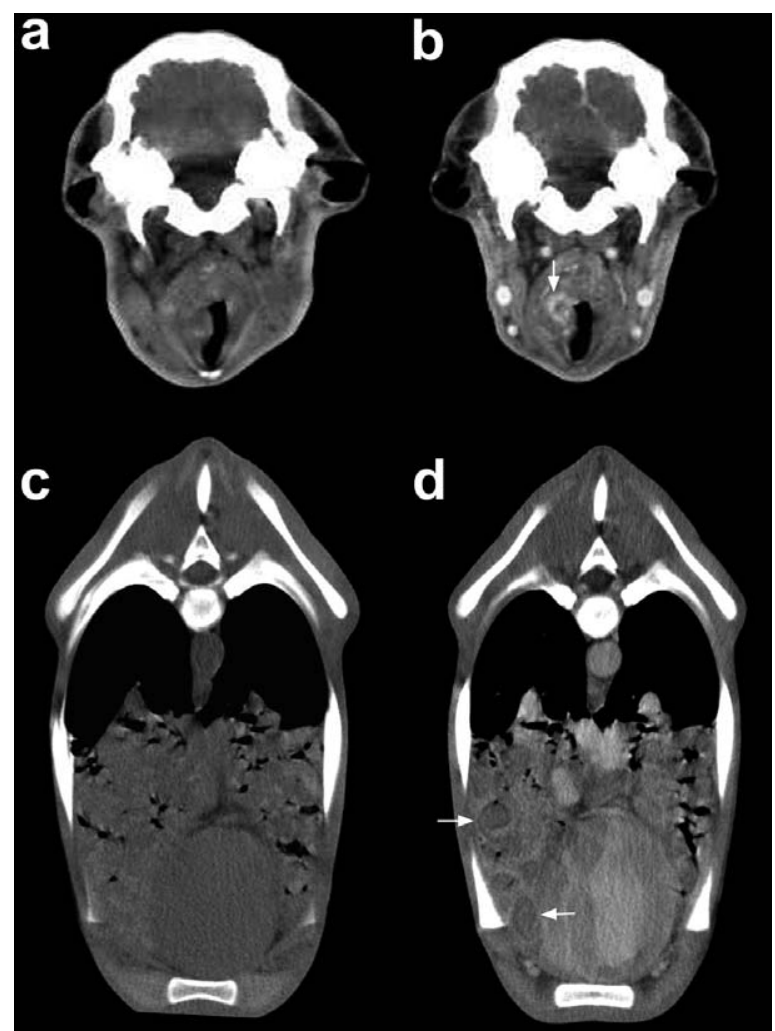

Fig. 3. Non-contrast (a, c) and post-contrast (b, d) transverse CT images in the calf with pharyngeal abscess and multiple pulmonary abscesses. Post-contrast CT images of the neck and lung fields show ring enhancement ( $b$ and $d$, arrows), indicating abscesses.

abscess. This decision was based on our clinical experience in bovine practice. Gross brain examination showed

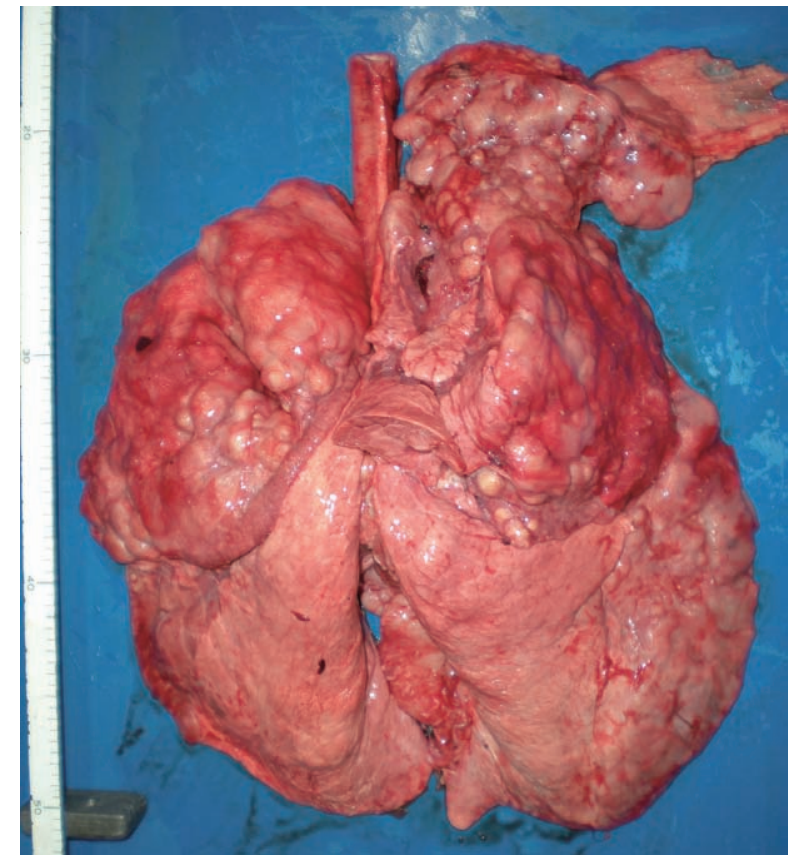

Fig. 4. Photograph of the lung in Case 2 showing the bulging nodules throughout the parenchymal surfaces.

cerebral edema and a well-delineated 1-cm sized mass, including opalescent fluid in the ventral region of the cerebellar vermis (Fig. 2). Bacteroides sp. was identified from the opalescent fluid by means of a microbiological study. Histopathological examination of the mass confirmed a cerebellar abscess surrounded by gliomesenchymal tissue.

Case 2 presented with a 2-month history of cough and dyspnea with no improvement when treated for pneumonia. The calf was treated with kanamycin $(1,250 \mathrm{mg}$, IM) for 1 week, and amoxicillin (750 mg, IM) for the following 1 week. Upon arrival, the calf was tachypneic with exertional dyspnea and stertorous inspiration. Tachycardia was also present. Thoracic auscultation revealed increased bilateral lung sounds. Nasopharyngeal endoscopy showed swelling and redness of the pharynx. The pharyngeal lumen was narrowed and obscured by the mass effect.

Hematological examination showed severe leukocytosis $(75,500 \mathrm{cells} / \mu \mathrm{L})$ with neutrophilia $(86 \%)$ and erythrocytosis $\left(13.6 \times 10^{6} / \mu \mathrm{L}\right)$. Blood gas analysis (OPTI CCA TS, Sysmex, Japan) revealed an increased partial pressure of carbon dioxide $(61.5 \mathrm{~mm} \mathrm{Hg}$, reference range $38-46 \mathrm{~mm} \mathrm{Hg}$ ) and decreased partial pressure of oxygen (48.0 $\mathrm{mm} \mathrm{Hg}$, reference range 77-92 $\mathrm{mm} \mathrm{Hg}$ ).

Lateral computed radiographs of the thorax revealed an extensive alveolar pattern and air bronchograms in the ventral part of the lung. Cervical and thoracic CT was performed for inspection of the pharynx and lung fields. The calf was positioned in sternal recumbency under general anesthesia, the same protocol as that of Case 1. Contiguous CT scans were obtained with $135 \mathrm{kV}, 150 \mathrm{~mA}$, and $3.0 \mathrm{~mm}$ 
slice thickness of the neck and thorax. In non-contrast CT with soft tissue window (WL 350, WW 70) of the neck, the right dorsal nasopharyngeal wall was displaced ventrally and to the left by a mass. Generalized thickening of the nasopharyngeal mucosa was present (Fig. 3a). Post-contrast CT images revealed a round mass $(4.6 \times 2.9 \mathrm{~cm})$ with ring enhancement in the right dorsal part of the pharynx (Fig. 3b, arrow). In the non-contrast CT of the thorax, multiple nodule-like lesions and consolidation isodense to the heart were detected in the ventral region of the lung lobes (Fig. 3c). Multifocal masses with ring enhancement in the lung lobes were seen on contrast-enhanced CT (Fig. 3d, arrows).

The calf was euthanized after the CT examination and necropsy was performed. On gross examination, opalescent caseous abscess was found at the right dorsal part of the pharynx. Gross pulmonary examination showed well-delineated, bulging pinpoint to $5-\mathrm{cm}$ nodules including opalescent caseous abscess scattered throughout the pleural and parenchymal surfaces of the entire lung field except dorsal part of the caudal lung lobes (Fig. 4). Severe adhesions of the pleura, diaphragm, and mediastinum were detected. Enlarged mediastinal lymph nodes were detected. Multiple pulmonary abscesses with pleuritis and pharyngeal abscess caused by Mycoplasma spp. was confirmed with histopathological examination and microbiological study.

The use of contrast medium is routinely recommended with CT to maximize detection of lesions and to provide further lesion characterization. The degree and pattern of enhancement are variable according to diseases [4]. A ring pattern of enhancement is detected in cases of abscess $[3,5]$, although ring enhancement has been reported with neoplastic and non-neoplastic brain lesions in dogs as well [8]. A ring is created by a zone of blood vessels, reactive astrocytes, and fibroblasts that surround a central core of necrosis due to various causes. The ring enhancement effect thus suggests that necrosis results from either a defective blood-brain barrier in this zone and/or retention of contrast material in the abnormal vascular tissue due to loss of autoregulation [8]. In this study, the ring patterns were detected at pharyngeal and pulmonary abscesses in Case 2 and cerebellar abscess in Case 1. El-Khodery et al. reported that large cerebral abscesses may be detected with non-contrast CT [2]. In Case 1, non-contrast CT missed a small cerebellar abscess and contrast-enhanced CT showed a lesion with ring pattern of enhancement. This result suggests that contrast-enhanced CT may be a useful technique for diagnosing small brain abscesses in cattle.

In Case 2, non-contrast CT revealed a mass in the pharynx and multiple nodule-like lesions in the lung fields. Despite the fact that non-contrast CT was helpful in identifying a mass causing the dyspnea, it had limited informative value as to the cause or exact location of such a mass. However, contrast-enhanced CT showed masses with a ring pattern of enhancement in the pharynx and lung fields. It was a helpful clue to identify the location of the mass and to diagnose abscesses.

Upper respiratory tract disease has rarely been reported as a cause of respiratory distress in calves [1]. There is only one case of retropharyngeal abscess diagnosed with magnetic resonance imaging [1]. To our knowledge, this study presents the first report of contrast-enhanced CT for detection of a pharyngeal abscess in a calf. Furthermore, CT findings of multiple pulmonary abscesses in cattle have not been reported previously. This report provides the first description of antemortem diagnosis of multiple pulmonary abscesses using contrast-enhanced CT.

In small animal practices, contrast-enhanced CT studies are routinely carried out. Although the diagnostic value of contrast-enhanced $\mathrm{CT}$ in production animals such as cattle may be identical, the reports on the use of contrast-enhanced $\mathrm{CT}$ in cattle are few [5]. The use of CT including contrastenhanced CT may enable veterinarians to diagnose disorders at an early stage, evaluate the prognosis, and assess when to slaughter cattle so that economic benefits are obtained. Furthermore, accumulation of CT data on cattle disease including abscesses is expected to be helpful for diagnosing diseases in production animals, which is currently difficult due to the lack of availability of adequate imaging data. This report demonstrated that contrastenhanced CT can be valuable in the diagnosis of abscesses in cattle.

\section{REFERENCES}

1. Buczinski, S., Fecteau, G., Alexander, K. and Norman-Carmel, E. 2008. Use of magnetic resonance imaging in the diagnosis of upper respiratory obstruction in a calf. Can. Vet. J. 49: 275279.

2. El-Khodery, S., Yamada, K., Aoki, D., Kamio, K., Kishimoto, M., Shimizu, J., Kobayashi, Y., Inokuma, H., Ishii, M., Yamauchi, S. and Matsui, T. 2008. Brain abscess in a Japanese black calf: Utility of computed tomography (CT). J. Vet. Med. Sci. 70: 727-730.

3. Gerros, T. C., Mattoon, J. S. and Snyder, S. P. 1998. Use of computed tomography in the diagnosis of a cerebral abscess in a goat. Vet. Radiol. Ultrasound 39: 322-324.

4. Kraft, S. L. and Gavin, P. R. 1999. Intrcaranial neoplasia. Clin. Tech. Small. Anim. Pract. 14: 112-123.

5. Lee, K., Yamada, K., Tsuneda, R., Kishimoto, M., Shimizu, J., Kobayashi, Y., Furuoka, H., Matsui, T., Sasaki, N., Ishii, M., Inokuma, H., Iwasaki, T. and Miyake, Y. 2009. Clinical experience of using multidetector-row CT for the diagnosis of disorders in cattle. Vet. Rec. 165: 559-562.

6. Schultz, R. M., Wisner, E. R., Johnson, E. G. and MacLeod, J. S. 2009. Contrast-enhanced computed tomography as a preoperative indicator of vascular invasion from adrenal masses in dogs. Vet. Radiol. Ultrasound 50: 625-629.

7. Shimizu, J., Yamada, K., Mochida, K., Kako, T., Muroya, N., Teratani, Y., Kishimoto, M., Lee, K., Iwasaki, T. and Miyake, Y. 2009. Comparison of the diagnosis of intervertebral disc herniation in dogs by CT before and after contrast enhancement of the subarachnoid space. Vet. Rec. 165: 200202.

8. Wolf, M., Peroia, V., Higgins R. J., Koblik, P. D., Turrel, J. M. and Owens, J. M. 1995. Intracranial ring enhancing lesions in dogs: a correlative CT scanning and neuropathologic study. Vet. Radiol. Ultrasound 36: 16-20. 